\title{
Bioresorbable vascular scaffolds in saphenous vein grafts (data from OCTOPUS registry)
}

\author{
Tomasz Roleder, Wojciech Wanha, Grzegorz Smolka, Jacek Zimoch, Andrzej Ochała, Wojciech Wojakowski \\ $3^{\text {rd }}$ Department of Cardiology, Medical University of Silesia, Katowice, Poland
}

Postep Kardiol Inter 2015; 11, 4 (42): 323-326 DOI: $10.5114 /$ pwki.2015.55604

\section{Introduction}

Percutaneous coronary intervention (PCI) of saphenous vein grafts (SVG) with metallic stents is associated with a high rate of restenosis (19-21\%) and target vessel failure in the long-term follow-up [1]. Potentially, implantation of a bioresorbable vascular scaffold (BVS) (ABSORB, Abbott Laboratories) into the stenotic SVG may be an alternative to metallic stents [2]. Although the effectiveness of BVS in treatment of de novo coronary lesions in native vessels is well documented [3], none of the previous studies evaluated the results of BVS implantation into SVG.

Recently, we reported an imaging follow-up of a case of successful BVS implantation to treat a de novo stenotic SVG lesion [4-6]. Therefore, in this report we present pilot results from the OCTOPUS registry of a subgroup of patients with significant de novo SVG stenosis treated with BVS implantation.

\section{Methods}

OCTOPUS is a single-center registry evaluating the morphology of SVG lesions and stent healing by multimodality imaging including optical coherence imaging (OCT) and assessing long-term clinical outcomes of SVG interventions in patients with stable coronary artery disease (CAD) and acute coronary syndromes (ACS). Here we focus on patients treated with BVS implantation into SVG. The study was approved by the Ethics Committee of the Medical University of Silesia and conforms to the Declaration of Helsinki. All of the patients were enrolled in the study only after the patient gave their informed written consent.

\section{Inclusion criteria}

The registry included patients with a history of coronary artery bypass grafting utilizing SVG with recurrent stable CAD or ACS. The study exclusion criteria were as follows: age < 18 years old, glomerular filtration rate less then $45 \mathrm{ml} / \mathrm{min} / 1.72 \mathrm{~m}^{2}$, severe valve disease warranting redo cardiac surgery and contrast allergy.

\section{Quantitative coronary angiography}

Quantitative angiography (QA) of SVG lesions was performed before and after BVS implantation using Horizon Cardiology 12.2 software (McKesson company) to evaluate reference lumen diameter (RLD), minimal lumen diameter (MLD), percentage diameter stenosis (\%DS), and lesion length. At follow-up, MLD, \%DS and late lumen loss (LLL) were evaluated. Late lumen loss was defined as the difference between the MLD immediately after the procedure and MLD at follow-up within the implanted BVS.

\section{Optical coherence tomography imaging}

Optical coherence tomography imaging was performed after the scaffold implantation and at the follow-up. The St Jude iLumien OPTIS Medical system was used for OCT imaging. The OCT probe (mid marker of the OCT Dragonfly catheter) was positioned $5 \mathrm{~mm}$ distally to the scaffold intended to be analyzed. All OCT imaging was performed using automated pullback triggered by the hand injection of contrast.

\section{Optical coherence tomography analysis}

CAAS IntraVascular software 1.1 (Pie Medical Imaging) was used for offline analysis of implanted BVS. The region of interest (ROI) was selected between proximal and distal edges of BVS where the OCT-visualized struts occupied more than $180^{\circ}$ of the lumen's circumference. The analysis was performed every $1 \mathrm{~mm}$ to measure minimal lumen area (MLA) and minimal scaffold area (MSA). Minimal scaffold area was measured the outer circumference of polymeric struts. The reference lumen

\section{Corresponding author:}

Tomasz Roleder MD, PhD or Wojciech Wojakowski MD, $3^{\text {rd }}$ Department of Cardiology, Medical University of Silesia, $45 / 47$ Ziolowa St, 40-635 Katowice, Poland, phone: +48 884096 034, fax: +48 3225239 30, e-mail: tomaszroleder@gmail.com

Received: 15.06.2015, accepted: 9.08.2015. 
area (RLA) was the average of the largest lumen area of two $5 \mathrm{~mm}$ long vessel segments located proximally and distally to the edges of BVS.

Polymeric strut apposition was also assessed, and if there was a gap between the polymeric strut and the vessel's lumen contour, malapposition was diagnosed [7].

At the follow-up, polymeric strut coverage by neointima was also assessed. The complete coverage of BVS by neointima was identified if 4 corners of the polymeric strut had lost the right angle shape with signs of tissue coverage [8].

\section{Statistical analysis}

The statistical analysis was performed using MedCalc Software 14.12.0. As it was a small group of patients, the results were presented as the median and range.

\section{Results}

\section{Patients' characteristics}

Six (4 male, 2 female) patients referred for $\mathrm{PCl}$ of de novo SVG lesions received BVS (Absorb, Abbott Laboratories). The median age was 73 years (range: 5783 years). All patients had hypertension and 3 patients had diabetes. The median time from coronary artery bypass grafting to BVS implantation was 13 years (range: 3-22 years). All patients received dual antiplatelet therapy for at least 6 months or 12 months if presenting with ACS.

\section{Procedural data}

The median diameter of implanted BVS was $3.25 \mathrm{~mm}$ (range: $2.5-3.5 \mathrm{~mm}$ ) with the median length $15 \mathrm{~mm}$ (range: $12-28 \mathrm{~mm}$ ). For all BVS implantations distal protection devices were used and all treated lesions were predilated with a semi-/non-compliant (NC) balloon according to the operator's choice. For postdilation NC balloons were used.

\section{Quantitative angiography analysis}

Two BVS were implanted into the SVG of each right coronary artery, left anterior descending artery and circumflex artery. Quantitative angiography analysis revealed the median RLD of $3.13 \mathrm{~mm}$ (range: 2.25$3.45 \mathrm{~mm}$ ) and median MLD of $1.12 \mathrm{~mm}$ (range: $0.52-$ $1.86 \mathrm{~mm}$ ) with median \%DS $=61 \%$ (range: $46-72 \%$ ). The $46 \%$ stenosis of one lesion was verified by OCT imaging, which revealed a $53 \%$ area stenosis with small MLA (RLA $=5.4 \mathrm{~mm}^{2}, \mathrm{MLA}=2.5 \mathrm{~mm}^{2}$ ). The median lesion length was $14.4 \mathrm{~mm}$ (range: 6.6-20.4 mm). Post-procedure MLD of BVS was $2.59 \mathrm{~mm}$ (range: $2.0-3.0 \mathrm{~mm}$ ) with median $\%$ DS $=13.5 \%$ (range: $8.1-16.7 \%$ ).

Four patients had angiography at 6-month followup and with median BVS MLD $=2.59 \mathrm{~mm}$ (range: 1.8 $2.78 \mathrm{~mm}$ ) and median \%DS = 14.1\% (range: $10.5-23 \%$ ).
Median LLL was $0.20 \mathrm{~mm}$ (range: $0.0-0.52 \mathrm{~mm}$ ) at 6-month follow-up. No SVG restenosis was found at the site of BVS implantation at 6-month follow-up. One patient also had 18-month angiographic follow-up, which presented BVS MLD $=2.38$ with $\mathrm{LLL}=0.24 \mathrm{~mm}$ and $\% \mathrm{DS}=22 \%$.

\section{Optical coherence tomography analysis}

Results of implantation were assessed by OCT in 3 BVS at baseline, in 3 BVS at 6-month follow-up, and 1 BVS 18 months after implantation. Two BVS had both OCT at baseline and OCT at 6-month follow-up.

\section{Baseline}

In total, $38 \mathrm{~mm}$ of BVS were analyzed at baseline. The RLA was $10.6 \mathrm{~mm}^{2}, 9.1 \mathrm{~mm}^{2}$ and $5.4 \mathrm{~mm}^{2}$, and MSA was $7.7 \mathrm{~mm}^{2}, 9.4 \mathrm{~mm}^{2}$ and $5.4 \mathrm{~mm}^{2}$, respectively. Twenty-three out of 151 polymeric struts (15\%) were malapposed in the first patient $(\mathrm{ROI}=11 \mathrm{~mm})$ at baseline and 1 of 125 polymeric struts was malapposed $(0.8 \%)$ in the second patient $(\mathrm{ROI}=16 \mathrm{~mm})$. The detected malappositions were not modified after BVS implantation. None of 111 polymeric struts was malapposed in the last patient $(\mathrm{ROI}=11 \mathrm{~mm})$. In one case a plaque protrusion and distal edge dissection were detected directly after implantation but without significant consequences (Figure 1). Thrombus was not found at baseline analysis.

\section{6-month follow-up}

Optical coherence tomography imaging of BVS at 6-month follow-up was performed in 3 patients with $\mathrm{ROI}=18 \mathrm{~mm}, \mathrm{ROI}=12 \mathrm{~mm}$ and $\mathrm{ROI}=22 \mathrm{~mm}$ at median 7 (5-7) months after the index procedure. The MSA at follow-up was $8.6 \mathrm{~mm}^{2}, 5.7 \mathrm{~mm}^{2}$ and $8.2 \mathrm{~mm}^{2}$, respectively. All BVS analyzed at 6-month follow-up were well apposed. In the 2 first patients from baseline analysis $86 \%(153 / 177)$, and $87 \%(99 / 125)$ of polymeric struts were covered by neointima respectively. In the BVS of the other patient, that was not analyzed at baseline, $88 \%$ $(25 / 202)$ of polymeric struts were covered by neointima at 6-month follow-up, even at the site of scaffold disruption (Figure 1).

\section{8-month follow-up}

Optical coherence tomography imaging at 18-month follow-up after implantation of BVS was performed only in 1 patient and showed that 97\% (5/175) of polymeric struts were covered by neointima with MLA $5.2 \mathrm{~mm}^{2}$ and MSA $8.3 \mathrm{~mm}^{2}$. At the site of BVS disruption that was identified 6 months after its implantation, a lipid-rich plaque protrusion was detected at 18-month follow-up (Figure 1). 

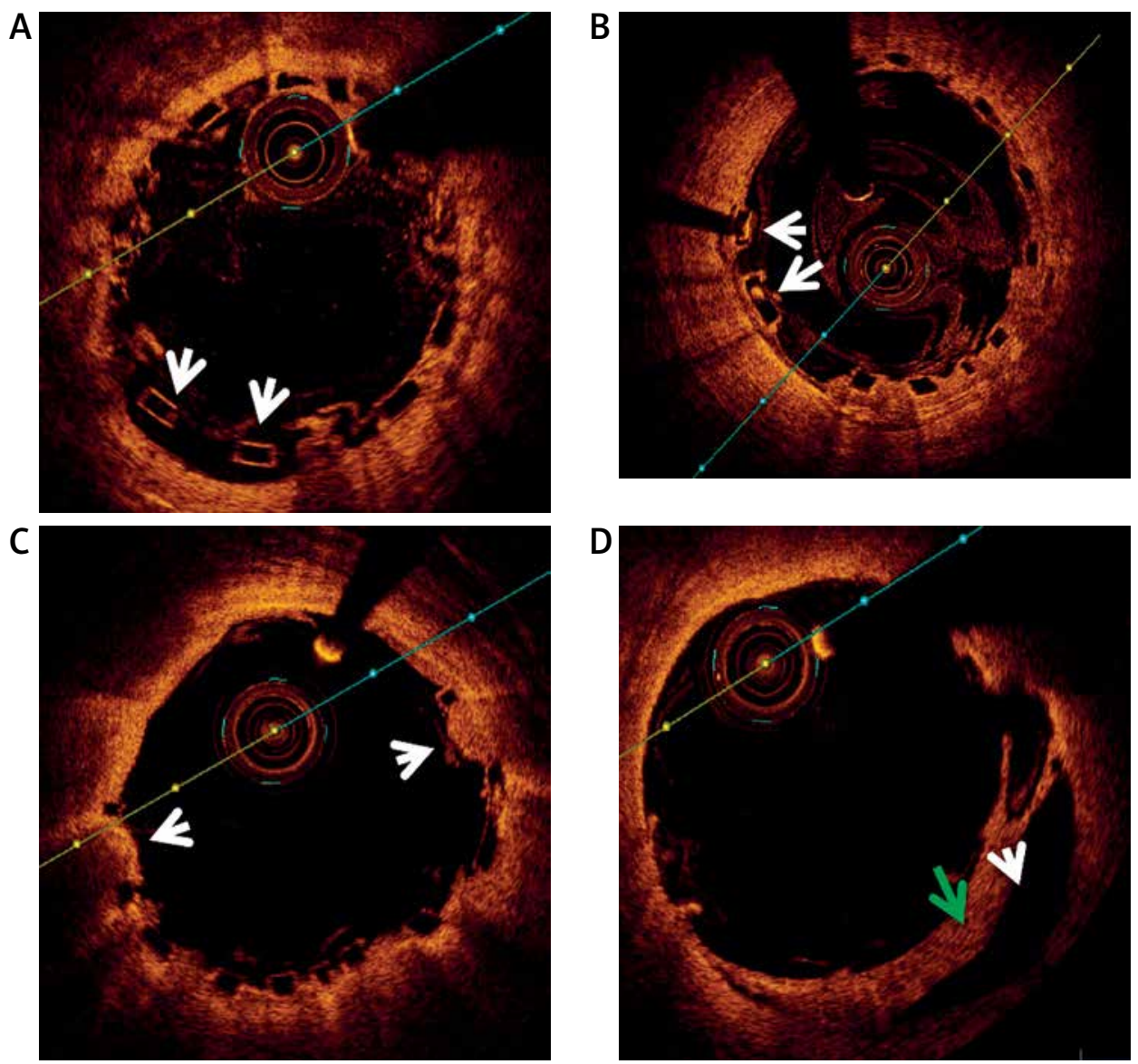

E
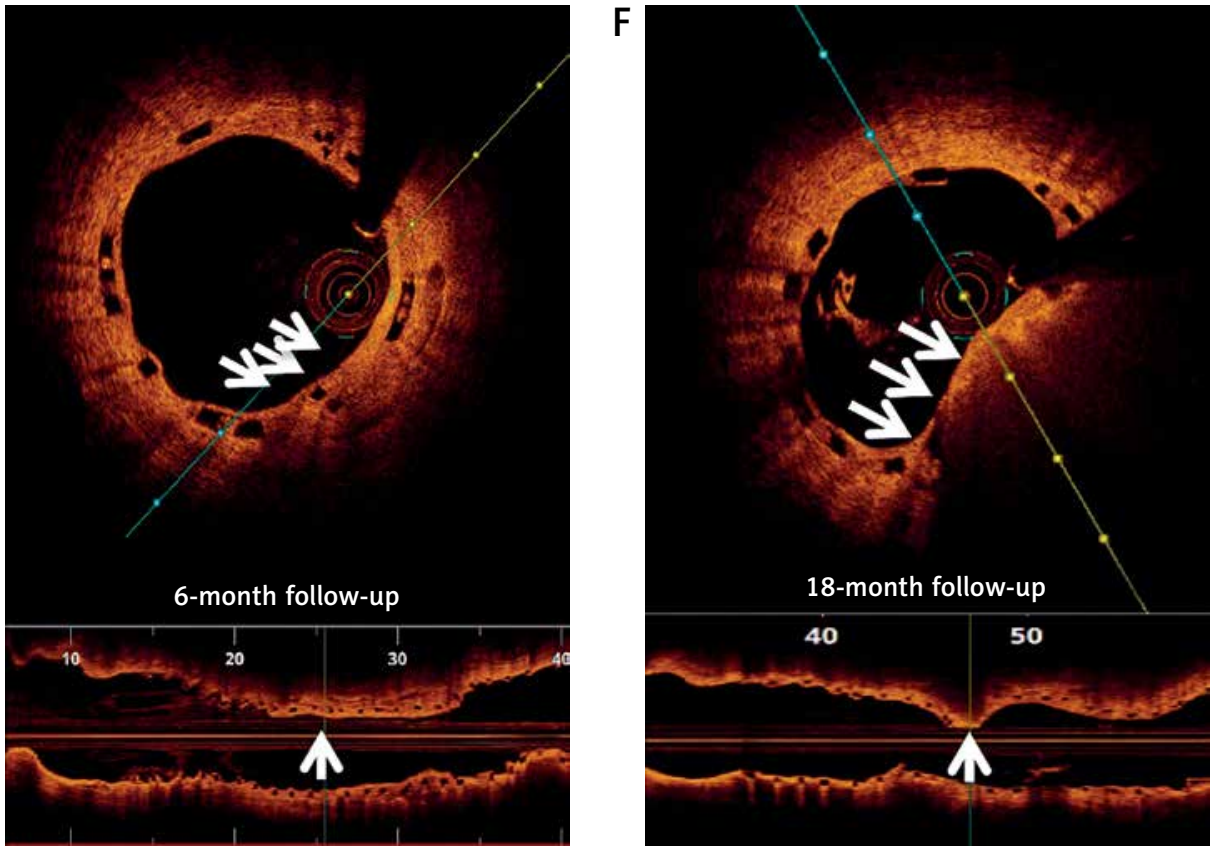

Figure 1. Representative optical coherence tomography images of ABSORB implanted into saphenous vein graft. A - Malapposed polymeric struts (arrows). B - Polymeric struts not covered by neointima at 6-month follow-up (arrows). C - Plaque protrusion through polymeric struts (arrows). D - Vessel's dissection (white arrow) after BVS implantation with visible intimal flap (green arrow). E and F - Present OCT cross-section and longitudinal images of the same BVS at 6 and 18 months after implantation into right coronary saphenous vein graft. E Two overhang polymeric struts covered by neointima (white arrows) 6 months after implantation. $\mathbf{F}-$ The same BVS 18 months after implantation with detected plaque protrusion at site of two overlapping polymeric struts 


\section{Patients' follow-up}

All patients were followed up to 12 months after BVS implantation. There were no deaths or target vessel revascularization defined as any revascularization within the treated vessel.

\section{Discussion}

Implantation of BVS is safe and feasible providing the diameter of the scaffold falls within the recommended limit of scaffold expansion. Therefore this approach is limited to conduits up to $4 \mathrm{~mm}$ in diameter. This report for the first time provides the OCT analysis of BVS healing in SVG. At the time of implantation, a similar ratio of malapposed polymeric struts was observed as in native coronary arteries, as previously reported [8]. However, the BVS coverage was less evident as compared to that observed in native coronary arteries at follow-up [7]. It is probably a similar phenomenon as in the metallic everolimus-eluting stent implanted into the SVG [9], which suggested that the degree of healing might be related to the particular anatomy and tissue properties of SVG. Moreover, the presence of atheroma that protruded through polymeric struts 18 months after implantation suggested that either neoatherosclerosis developed or the mechanical properties of the graft changed the geometry of the ROI [10]. It warrants further large clinical studies to investigate the safety and efficacy of ABSORB implantation into the SVG.

\section{Conclusions}

This report presents long-term imaging data on a small number of patients with SVG stenosis treated with BVS. It suggests that the healing of BVS in SVG is not impaired, but a properly sized clinical trial is needed to confirm this.

\section{Conflict of interest}

The authors declare no conflict of interest.

\section{Funding}

This work was supported by European Union structural funds (Innovative Economy Operational Program POIG.01.01.02-00-109/09-00) and statutory funds of the Medical University of Silesia.

\section{References}

1. Nair S, Fath-Ordoubadi F, Clarke B, et al. Late outcomes of drug eluting and bare metal stents in saphenous vein graft percutaneous coronary intervention. Eurolntervention 2011; 6: 985-91.

2. Lesiak M, Araszkiewicz A. "Leaving nothing behind": is the bioresorbable vascular scaffold a new hope for patients with coronary artery disease? Postep Kardiol Inter 2014; 10: 283-8.
3. Dudek D, Rzeszutko L, Zasada W, et al. Bioresorbable vascular scaffolds in patients with acute coronary syndromes: the polar acs study. Pol Arch Med Wewn 2014; 124: 669-77.

4. Ong PJ, Jafary FH, Ho HH. "First-in-man" use of bioresorbable vascular scaffold in saphenous vein graft. Eurolntervention 2013; 9: 165.

5. Roleder T, Parma Z, Smolka G, et al. Optical coherence tomography imaging of everolimus-eluting bioresorbable vascular scaffold implanted into coronary vein graft at 3-month follow-up. Eur Heart J 2014; 35: 2207.

6. Roleder T, Ochala A, Smolka G, et al. Implantation of a bioabsorbable vascular scaffold into a coronary vein graft: a twoweek angiography follow-up. Kardiol Pol 2014; 72: 281.

7. Gutierrez-Chico JL, Gijsen F, Regar E, et al. Differences in neointimal thickness between the adluminal and the abluminal sides of malapposed and side-branch struts in a polylactide bioresorbable scaffold: evidence in vivo about the abluminal healing process. JACC Cardiovasc Interv 2012; 5: 428-35.

8. Gomez-Lara J, Radu M, Brugaletta $S$, et al. Serial analysis of the malapposed and uncovered struts of the new generation of everolimus-eluting bioresorbable scaffold with optical coherence tomography. JACC Cardiovasc Interv 2011; 4: 992-1001.

9. Papayannis AC, Michael TT, Yangirova D, et al. Optical coherence tomography analysis of the stenting of saphenous vein graft (sos) Xcience V Study: use of the everolimus-eluting stent in saphenous vein graft lesions. J Invasive Cardiol 2012; 24: 390-4.

10. Ali ZA, Roleder T, Narula J, et al. Increased thin-cap neoatheroma and periprocedural myocardial infarction in drug-eluting stent restenosis: multimodality intravascular imaging of drug-eluting and bare-metal stents. Circ Cardiovasc Interv 2013; 6: 507-17. 